\title{
COMPLEXITY OF PARTIAL INVERSE ASSIGNMENT PROBLEM AND PARTIAL INVERSE CUT PROBLEM*
}

\author{
XiaOguANG YANG $^{1}$ \\ Communicated by Ph. Chrétienne
}

\begin{abstract}
For a given partial solution, the partial inverse problem is to modify the coefficients such that there is a full solution containing the partial solution, while the full solution becomes optimal under new coefficients, and the total modification is minimum. In this paper, we show that the partial inverse assignment problem and the partial inverse minimum cut problem are NP-hard if there are bound constraints on the changes of coefficients.
\end{abstract}

Keywords: Partial inverse assignment problem, partial inverse minimum cut problem, NP-hard.

Mathematics Subject Classification. 68Q25, 90B10, 90C27.

\section{INTRODUCTION}

An inverse optimization problem is to modify the coefficients of the objective function optimally, such that a given solution becomes an optimal solution under new coefficients. Due to its theoretical and practical importance, the inverse problem has attracted much attention. It has been shown that many inverse combinatorial optimization problems can be solved by combinatorial strongly polynomial algorithms [1-5]. Since the given solution is a full solution of the original

Received February, 2000. Accepted January, 2001.

* Research is partially supported by National Natural Science Foundation of China (No. 79790130) and National $863 \mathrm{Hi}$-Tech Project (No. 863-306-ZT04-04-1).

${ }^{1}$ Laboratory of Management, Decision and Information Systems, Institute of Systems Science,

Chinese Academy of Sciences, Beijing 100080, P.R. China; e-mail: xgyang@iss04.iss.ac.cn

(C) EDP Sciences 2001 
optimization problem, we may refer the inverse problems defined above as inverse problems with full given solution.

But in reality, we may know more about a partial solution than a full solution. Sometimes what we are most interested in is how to guarantee that a partial solution is contained in an optimal solution. The partial inverse problem is to modify coefficients optimally such that there exists a full solution containing a given partial solution which becomes optimal under new coefficients. We have shown that several partial inverse network flow problems can be solved efficiently if there are no constraints on the modification of coefficients [6]. In this paper, we prove that the partial inverse assignment problem and the partial inverse minimum cut problem are NP-hard if there are bound constraints on the modification of coefficients.

\section{Partial inverse ASSignMent PROBLEM}

Let $U$ and $V$ be two node sets such that $|U|=|V|, E=\{(u, v) \mid u \in U, v \in V\}$. Let $c$ be a weight function defined on $E$. The minimum assignment is to find a subset $M \subset E$ such that each node is linked by one and only one edge in $M$ (that is, the nodes of $U$ and $V$ are connected pair-wise, and $|M|=|U|=|V|$ ) and $\sum_{(u, v) \in M} c(u, v)$ is minimum.

Given an assignment $M$, the inverse assignment problem is to modify the weight function $c$ to $c^{*}$ such that $M$ is minimum with respect to $c^{*}$ and the total modification is minimum.

It has been shown that the inverse assignment problem with various cost forms and bound constraints on the modification can be solved by combinatorial strongly polynomial algorithms $[2-4,10]$.

Now suppose that only a partial assignment $P$ is given (each node is linked by at most one edge in $P$, and $|P| \leq|U|)$. Let $b$ be a bound function defined on $E$. The partial inverse assignment problem is to modify the weight function $c$ to $c^{*}$ such that

(a) there is an assignment $M^{*}$ covering $P$ which is minimum with respect to $c^{*}$;

(b) $\left|c(e)-c^{*}(e)\right| \leq b(e), \forall e \in E$ and;

(c) the total modification $\sum_{e \in E}\left|c(e)-c^{*}(e)\right|$ is minimum.

If there are not bound constraints, we have proved that the inverse partial assignment problem can be solved by a combinatorial strongly polynomial algorithm [6]. Here we will show that the inverse problem turns to be NP-hard when the bound constraints are imposed.

It is clear that the partial inverse assignment is in NP-class. Therefore, to prove the NP-hardness, we need only prove that the decision version of the partial inverse assignment problem can be reduced to a NP-complete problem, even-odd partition problem [7]. 


\section{EVEN-ODD PARTITION PROBLEM}

Given a set of $2 n$ positive integers $\left\{a_{1}, a_{2}, \cdots, a_{2 n}\right\}$ such that $a_{i}>a_{i+1}$ for each $1 \leq i<2 n$, is there a partition of $I=\{1,2, \cdots, 2 n\}$ into two subsets $S$ and $I \backslash S$ such that, for each $1 \leq i \leq n, S$ (and hence $I \backslash S$ ) contains exactly one of $\{2 i-1,2 i\}$. (We call such subset $S$ as even-odd subset.), and $\sum_{i \in S} a_{i}=\sum_{i \in I \backslash S} a_{i}$ ? If such an even-odd subset $S$ exists, we call $S$ a feasible even-odd partition set.

Let $B=\frac{1}{2} \sum_{i=1}^{2 n} a_{i}$. Without loss of generality, we suppose that $B$ is an integer and $B \geq a_{i}$ for all $1 \leq i \leq 2 n$.

Theorem 1. The partial inverse assignment problem is NP-hard if there are bound constraints.

Proof. Let $a_{1}>a_{2}>\cdots>a_{2 n}$ and $B$ be an instance of even-odd partition problem.

When $n=1$ or 2 , it is trivial to verify whether a given instance of even-odd partition problem has a feasible solution. Thus we only consider arbitrarily large $n \geq 3$.

We construct the following partial inverse assignment problem. Let $U=\left\{u_{1}, u_{2}\right.$, $\left.\ldots, u_{2 n}\right\}$ and $V=\left\{v_{1}, v_{2}, \ldots, v_{2 n}\right\}$. Let us decompose the edge set into the following five subsets.

$$
\begin{aligned}
& E_{1}=\left\{\left(u_{i}, v_{i}\right) \mid i=1,2, \ldots, 2 n\right\} \\
& \left.E_{2}=\left\{u_{2 i-1}, v_{2 i}\right) \mid i=1,2, \ldots, n\right\} \\
& \left.E_{3}=\left\{u_{2 i}, v_{[2 i+1]}\right) \mid i=1,2, \ldots, n\right\} \\
& \left.E_{4}=\left\{u_{2 i}, v_{[2 k+1]}\right) \mid 1 \leq i \neq k \leq n\right\} \\
& E_{5}=\{\text { all other edges }\}
\end{aligned}
$$

Where $[2 i+1]=2 i+1$ if $2 i+1<2 n$ and $[2 n+1]=1$.

Set the weight function $c$ as

$$
\begin{aligned}
c\left(u_{i}, v_{i}\right) & =B-\frac{1}{4 n} B & \left(u_{i}, v_{i}\right) & \in E_{1} \\
c\left(u_{2 i-1}, v_{2 i}\right) & =B & \left(u_{2 i-1}, v_{2 i}\right) & \in E_{2} \\
c\left(u_{2 i}, v_{[2 i+1]}\right) & =B+\frac{1}{2} a_{2 i-1} & \left(u_{2 i}, v_{[2 i+1]}\right) & \in E_{3} \\
c\left(u_{2 i}, v_{[2 k+1]}\right) & =B+\frac{1}{2} a_{2 i} & \left(u_{2 i}, v_{[2 k+1]}\right) & \in E_{4} \\
c(u, v) & =4 n B & (u, v) & \in E_{5} .
\end{aligned}
$$

Define the bound function $b$ as follows:

$$
\begin{aligned}
b\left(u_{2 i}, v_{[2 i+1]}\right) & =a_{2 i-1} & & \left(u_{2 i}, v_{[2 i+1]}\right) & \in & E_{3} \\
b\left(u_{2 i}, v_{[2 k+1]}\right) & =a_{2 i} & & \left(u_{2 i}, v_{[2 k+1]}\right) & \in & E_{4} \\
b(u, v) & =0 & & \text { all other edges. } & &
\end{aligned}
$$

Let $P=E_{2}=\left\{\left(u_{2 i-1}, v_{2 i}\right) \mid i=1,2, \ldots, n\right\}$. We claim that there exists a feasible even-odd partition if and only if there exists an assignment covering $P$ which 
is a minimum assignment with respect to a modified weight function $c^{*}$ such that $\left|c(e)-c^{*}(e)\right| \leq b(e), \forall e \in E$, and $\sum_{u \in U, v \in V}\left|c(u, v)-c^{*}(u, v)\right| \leq B$.

Obviously, the minimum assignment with respect to $c$ is $M=E_{1}=\left\{\left(u_{i}, v_{i}\right) \mid i\right.$ $=1,2, \ldots, 2 n\}$, and the total weight of $M$ with respect to $c$ is $\sum_{(u, v) \in M} c(u, v)$ $=2 n B-\frac{1}{2} B$.

Suppose that there is an assignment $M^{*}$ covering $P$ which becomes a minimum assignment with respect to a modified weight function $c^{*}$ such that $\left|c(e)-c^{*}(e)\right|$ $\leq b(e), \forall e \in E$ and $\sum_{u \in U, v \in V}\left|c(u, v)-c^{*}(u, v)\right| \leq B$.

We know that:

(1) since $b(u, v)=0$ for $(u, v) \in M$, we have $c^{*}(u, v)=c(u, v)$ for $(u, v) \in M$, and then $\sum_{(u, v) \in M} c^{*}(u, v)=2 n B-\frac{1}{2} B$. Thus we have

$$
\sum_{(u, v) \in M^{*}} c^{*}(u, v) \leq 2 n B-\frac{1}{2} B
$$

since $M^{*}$ is minimum with respect to $c^{*}$;

(2) since $P \subset M^{*}$, and each edge in $E_{1}$ is incident to an edge from $P$, no edge in $E_{1}$ is in $M^{*}$, i.e., $E_{1} \cap M^{*}=\emptyset$.

(3) since $c(u, v)=4 n B$ and $b(u, v)=0$ for $(u, v) \in E_{5}$, no edge in $E_{5}$ is in $M^{*}$, i.e., $E_{5} \cap M^{*}=\emptyset$.

Let $M^{\prime}=M^{*} \backslash P$. Then $M^{\prime}$ is a perfect matching between $U^{\prime}=\left\{u_{2}, u_{4}, \ldots, u_{2 n}\right\}$ and $V^{\prime}=\left\{v_{1}, v_{3}, \ldots, v_{2 n-1}\right\}$ with respect to edge set $E^{\prime}=E_{3} \cup E_{4}$.

Denote $S=\left\{2 i-1 \mid\left(u_{2 i}, v_{[2 i+1]}\right) \in M^{*} \cap E_{3}\right\} \cup\left\{2 i \mid\left(u_{2 i}, v_{[2 k+1]}\right) \in M^{*} \cap E_{4}\right\}$. Since there is exact one edge in $M^{*}$ linking to $u_{2 i}, S$ contains one and only one item of $\{2 i-1,2 i\}$. Therefore $S$ is an even-odd partition set.

Denote $x_{2 i-1}=c\left(u_{2 i}, v_{[2 i+1]}\right)-c^{*}\left(u_{2 i}, v_{[2 i+1]}\right)$ for $\left(u_{2 i}, v_{[2 i+1]}\right) \in M^{*} \cap E_{3}$, and $x_{2 i}=c\left(u_{2 i}, v_{[2 k+1]}\right)-c^{*}\left(u_{2 i}, v_{[2 k+1]}\right)$ for $\left(u_{2 i}, v_{[2 k+1]}\right) \in M^{*} \cap E_{4}$. We have $\sum_{(u, v) \in M^{*}} c(u, v)=2 n B+\frac{1}{2} \sum_{j \in S} a_{j}$, and then $\sum_{(u, v) \in M^{*}} c^{*}(u, v)=2 n B+\frac{1}{2} \sum_{j \in S} a_{j}$ $-\sum_{j \in S} x_{j}$.

From (1), we get

$$
\sum_{j \in S} x_{j} \geq \frac{1}{2} \sum_{j \in S} a_{j}+\frac{1}{2} B
$$

By bound constraints, we have

$$
\sum_{j \in S} x_{j} \leq \sum_{j \in S} a_{j} .
$$


By the budget constraint, we have

$$
\sum_{j \in S} x_{j} \leq B
$$

Combining (2) and (3), we have $\sum_{j \in S} a_{j} \geq B$. Combining (2) and (4), we have $\sum_{j \in S} a_{j} \leq B$. Therefore we obtain $\sum_{j \in S} a_{j}=B$, that is, $S$ is a feasible even-odd partition set.

Conversely, let $S$ be a feasible even-odd partition set. Denote $S_{e}$ the set of even numbers in $S$, and $S_{o}$ the set of odd numbers in $S$.

We have

$$
|S|=\left|S_{e}\right|+\left|S_{o}\right|=n .
$$

We claim that both $S_{e}$ and $S_{o}$ can not be empty sets. Otherwise there must be $S=\{1,3, \cdots, 2 n-1\}$ or $S=\{2,4, \cdots, 2 n\}$. Since $a_{1}>a_{2}>\cdots>a_{2 n}$, $\sum_{i=1}^{n} a_{2 i-1}>\sum_{i=1}^{n} a_{2 i}, S$ can not be a feasible even-odd partition set.

Since $n \geq 3$, at least one of $S_{e}$ and $S_{o}$ has two or more numbers.

Without loss of generality, assume that $\left|S_{e}\right| \geq 2$, let $E_{3}^{-}=\left\{\left(u_{2 i}, v_{[2 i+1]}\right)\right.$ $\left.\in E_{3} \mid 2 i-1 \in S_{o}\right\}$. Let $U^{-}=U^{\prime} \backslash\left\{u_{2 i} \mid\left(u_{2 i}, v_{[2 i+1]}\right) \in E_{3}^{-}\right\}, V^{-}=V^{\prime} \backslash\left\{v_{[2 i+1]} \mid\left(u_{2 i}\right.\right.$, $\left.\left.v_{[2 i+1]}\right) \in E_{3}^{-}\right\}$. Then $\left|U^{-}\right|=\left|V^{-}\right|=\left|S_{e}\right| \geq 2$.

(If $\left|S_{e}\right|=1$, we have $\left|S_{o}\right| \geq 2$. Then we define $E_{3}^{-}=\left\{\left(u_{2 i}, v_{[2 i+1]}\right) \mid 2 i \in S_{e}\right\}$, and define $U^{-}, V^{-}$according to $E_{3}^{-}$.)

Let $E^{-}=\left\{\left(u_{2 i}, v_{[2 k+1]}\right) \mid u_{2 i} \in U^{-}, v_{[2 k+1]} \in V^{-}, k \neq i\right\}$. It is easily seen that $E^{-} \subset E_{4}$.

Denote $M^{-}=\left\{\left(u_{2 i}, v_{[2 i+1]}\right) \mid u_{2 i} \in U^{-}\right\}$. Then $M^{-}$is a perfect matching between $U^{-}$and $V^{-}$, and $\left(U^{-} \cup V^{-}, E^{-} \cup M^{-}\right)$is indeed a complete bipartite graph.

Let $E_{4}^{-}$be a perfect matching of bipartite graph $\left(U^{-} \cup V^{-}, E^{-}\right)$. (It is easy to see that such a matching exists.)

Let us construct an assignment $M^{*}$ as follows

$$
M^{*}=E_{2} \cup E_{3}^{-} \cup E_{4}^{-} .
$$

Define a modified weight function as

$$
\begin{array}{lc}
c^{*}\left(u_{2 i}, v_{[2 i+1]}\right)=B-\frac{1}{2} a_{2 i-1} & \left(u_{2 i}, v_{[2 i+1]}\right) \in E_{3}^{-} \\
c^{*}\left(u_{2 i}, v_{[2 k+1]}\right)=B-\frac{1}{2} a_{2 i} & \left(u_{2 i}, v_{[2 k+1]}\right) \in E_{4}^{-} \\
c^{*}(u, v)=c(u, v) & \text { all other edges. }
\end{array}
$$

It is straightforward to verify that $\left|c(e)-c^{*}(e)\right| \leq b(e), \forall e \in E, \sum_{u \in U, v \in V} \mid c(u, v)$ $-c^{*}(u, v) \mid=B$, hence the bound constraints and budget constraint are satisfied.

Notice that, $E_{1} \cup E_{2} \cup E_{3}^{-} \cup E_{4}^{-}$is the set of $4 n$ lightest edges with respect to $c^{*}$, and it is an union of cycles covering every vertex of graph $(U \cup V, E)$ with $2 n$ vertices. Moreover $E_{1}$ and $M^{*}$ are only two perfect matchings contained 
in $E_{1} \cup E_{2} \cup E_{3}^{-} \cup E_{4}^{-}$, and $\sum_{e \in E_{1}} c^{*}(e)=\sum_{e \in M^{*}} c^{*}(e)=2 n B-\frac{1}{2} B$. Therefore $M^{*}$ is a minimum assignment with respect to $c^{*}$ which covers $P=E_{2}$.

Remark. When $n=2$, we can simply re-define the weights on the graph $(U \cup V, E)$, and check directly that a feasible even-odd partition set is corresponding to a minimum assignment under new weights with given partial assignment and required budget constraint.

In fact, let us set

$$
\begin{aligned}
c\left(u_{i}, v_{i}\right) & =\frac{7}{8} B \quad i=1,2,3,4 \\
c\left(u_{1}, v_{2}\right) & =c\left(u_{3}, v_{4}\right)=B \\
c\left(u_{2}, v_{1}\right) & =B+\frac{1}{2} a_{1} \\
c\left(u_{2}, v_{3}\right) & =B+\frac{1}{2} a_{2} \\
c\left(u_{4}, v_{1}\right) & =B+\frac{1}{2} a_{3} \\
c\left(u_{4}, v_{3}\right) & =B+\frac{1}{2} a_{4} \\
c(u, v) & =8 B \text { for all other edges }
\end{aligned}
$$

and

$$
\begin{aligned}
b\left(u_{2}, v_{1}\right) & =a_{1} \\
b\left(u_{2}, v_{3}\right) & =a_{2} \\
b\left(u_{4}, v_{1}\right) & =a_{3} \\
b\left(u_{4}, v_{3}\right) & =a_{4} \\
b(u, v) & =0 \text { for all other edges. }
\end{aligned}
$$

There are two perfect matchings contains $P=\left\{\left(u_{1}, v_{2}\right),\left(u_{3}, v_{4}\right)\right\}$, namely $M_{1}$ $=P \cup\left\{\left(u_{2}, v_{1}\right),\left(u_{4}, v_{3}\right)\right\}$ and $M_{2}=P \cup\left\{\left(u_{2}, v_{3}\right),\left(u_{4}, v_{1}\right)\right\}$.

If there exists a feasible even-odd partition set $S$, say $S=\{1,4\}$ and $a_{1}+a_{4}=B$, we can modify $c\left(u_{2}, v_{1}\right)$ and $c\left(u_{4}, v_{3}\right)$ as $c^{*}\left(u_{2}, v_{1}\right)=B$ $-\frac{1}{2} a_{1}$ and $c^{*}\left(u_{4}, v_{3}\right)=B-\frac{1}{2} a_{4}$, and keep weights of other edges unchanged. It is straightforward to check that $M_{1}$ becomes a minimum assignment with respect to a modified weight function $c^{*}$ such that $\left|c(e)-c^{*}(e)\right| \leq b(e), \forall e \in E$, and $\sum_{u \in U, v \in V}\left|c(u, v)-c^{*}(u, v)\right| \leq B$.

Conversely, if there exists an assignment covering $P$ which is a minimum assignment with respect to a modified weight function $c^{*}$ such that $\left|c(e)-c^{*}(e)\right|$ $\leq b(e), \forall e \in E$, and $\sum_{u \in U, v \in V}\left|c(u, v)-c^{*}(u, v)\right| \leq B$, we may assume the assignment is $M_{2}$.

Notice that $E_{1}=\left\{\left(u_{1}, v_{1}\right),\left(u_{2}, v_{2}\right),\left(u_{3}, v_{3}\right),\left(u_{4}, v_{4}\right)\right\}$ is an assignment of $(U \cup V, E)$, and we can not change weights on $E_{1}$, we have

$$
\sum_{(u, v) \in M_{2}} c^{*}(u, v) \leq \sum_{(u, v) \in E_{1}} c(u, v)=\frac{7}{2} B
$$

It is not difficult to deduce that $c^{*}\left(u_{2}, v_{3}\right)=B-\frac{1}{2} a_{2}$ and $c^{*}\left(u_{4}, v_{1}\right)=B-\frac{1}{2} a_{3}$ and $a_{2}+a_{3}=B$. Therefore $S=\{2,3\}$ is a feasible even-odd partition set. 


\section{Partial inVERSE Minimum CUt PROBlem}

Let $G=(V, A)$ be a directed graph, $s, t$ be two specific vertices of $V$. Let $c$ be a capacity function defined on $A$. Let $W$ be a subset of $V$ such that $s \in W$ but $t \notin W$, and denote $\bar{W}=V \backslash W$, and $(W, \bar{W})=\{(u, v) \mid u \in W, v \in \bar{W}\}$. Then $(W, \bar{W})$ is called a cut of $G$. The capacity of $(W, \bar{W})$ is defined as $c(W, \bar{W})$ $=\quad \sum c(u, v)$. A cut is called a minimum cut if its capacity is minimum. $(u, v) \in(W, \bar{W})$

Min-cut and Max-flow theorem is a fundamental work in combinatorial optimization.

Given a cut $(W, \bar{W})$, the inverse minimum cut problem is to modify the capacity function $c$ to $c^{*}$ such that $(W, \bar{W})$ is minimum with respect to $c^{*}$ and the total modification is minimum.

It has been shown that the inverse minimum cut problem with various cost forms and bound constraints on modification can be solved by combinatorial strongly polynomial algorithms $[8,9]$.

Now suppose that there are two subsets $S \subset V$ and $T \subset V$ such that $s \in S$, $t \in T$ and $S \cap T=\emptyset$. Let $b$ be a bound function defined on $A$. The partial inverse minimum cut problem is to modify the capacity function $c$ to $c^{*}$ such that:

(a) there is a cut $(W, \bar{W})$ satisfying $S \subset W, T \subset \bar{W}$, (we may call $(W, \bar{W})$ covering $(S, T))$, and $(W, \bar{W})$ is minimum with respect to $c^{*}$;

(b) $\left|c(e)-c^{*}(e)\right| \leq b(e), \forall e \in A$ and;

(c) the total modification $\sum_{e \in A}\left|c(e)-c^{*}(e)\right|$ is minimum.

As the inverse partial assignment problem, the inverse minimum cut problem has been shown to be "easy" problem [6] if there are not bound constraints. Now we show that the inverse problem becomes NP-hard when the bound constraints are imposed. To this end, let us introduce a variant of partition problem as follows:

Let $a_{1}, a_{2}, \ldots, a_{2 n}$ and $B$ be $2 n+1$ positive integers, such that $\sum_{i=1}^{2 n} a_{i}=2 B$, does there exist $I \subset\{1,2, \ldots, 2 n\}$ so as to $\sum_{i \in I} a_{i}=B$ ?

Since the number of item $a_{i}$ s is $2 n$, we may call this partition problem an evenitem partition problem. It is easy to show that the even-item partition problem is NP-complete.

In fact, let $\left\{c_{1}, c_{2}, \ldots, c_{n}\right.$ and $\left.D\right\}$ be an instance of partition problem. Without loss of generality, we assume that $D>1$. Let us construct an instance of even-item partition as: $a_{i}=c_{i}, a_{n+i}=D^{2} c_{i}, i=1,2, \ldots, n$, and $B=D+D^{3}$.

If there exists $I^{\prime} \subset\{1,2, \ldots, n\}$ such that $\sum_{i \in I^{\prime}} c_{i}=D$, setting $I=I^{\prime} \cup\{i+n \mid i$ $\left.\in I^{\prime}\right\}$, we can obtain $\sum_{i \in I} a_{i}=B$.

Conversely if there exists $I \subset\{1,2, \ldots, 2 n\}$ such that $\sum_{i \in I} a_{i}=B$, let us decompose $I=I^{\prime} \cup I^{\prime \prime}$ such that $I^{\prime} \subset\{1,2, \ldots, n\}$ and $I^{\prime \prime} \subset\{n+1, n+2, \ldots, 2 n\}$. Then $\sum_{i \in I} a_{i}=\sum_{i \in I^{\prime}} c_{i}+\sum_{i+n \in I^{\prime \prime}} D^{2} c_{i}$. 
We claim that $\sum_{i+n \in I^{\prime \prime}} c_{i}=D$. Otherwise, suppose that $\sum_{i+n \in I^{\prime \prime}} c_{i}>D$ (or $\sum_{i+n \in I^{\prime \prime}} c_{i}<D$ ), then $\sum_{i+n \in I^{\prime \prime}} c_{i} \geq D+1$ (or $\sum_{i+n \in I^{\prime \prime}} c_{i} \leq D-1$ ) since all numbers involved are positive integers. Therefore, $\sum_{i+n \in I^{\prime \prime}} D^{2} c_{i} \geq D^{2}(D+1)>D+D^{3}$ (or $\left.\sum_{i+n \in I^{\prime \prime}} D^{2} c_{i}<D^{3}-D\right)$. This contradicts $\sum_{i \in I} a_{i}=B=D+D^{3}$ since $0 \leq \sum_{i \in I^{\prime}} c_{i}$ $\leq 2 D$.

Now we can get $\sum_{i \in I^{\prime}} c_{i}=B-\sum_{i+n \in I^{\prime \prime}} D^{2} c_{i}=D$.

The equivalence between the partition problem and the even-item partition problem shows that the even-item partition is NP-complete.

It is clear that the partial inverse minimum cut problem is in NP-class. Therefore, to prove the NP-hardness, it is sufficient to prove that the decision problem of a partial inverse minimum cut problem can be reduced to an even-item partition problem.

Theorem 2. The partial inverse minimum cut problem with bound constraints is NP-hard.

Proof. Let $a_{1}, a_{2}, \ldots, a_{2 n}$ and $B$ be an instance of even-item partition problem. We construct the following partial inverse cut problem.

$$
\begin{aligned}
V & =\{s, t\} \cup\left\{u_{i}, v_{i}, w_{i} \mid i=1,2, \ldots, n\right\} \\
A & =\left\{\left(s, u_{i}\right),\left(u_{i}, v_{i}\right),\left(u_{i}, w_{i}\right),\left(v_{i}, t\right),\left(w_{i}, t\right) \mid i=1,2, \ldots, n\right\} .
\end{aligned}
$$

Define the capacity function as

$$
\begin{array}{rlrl}
c\left(s, u_{i}\right) & =2 B-\frac{1}{2 n} B & i & =1,2, \ldots, n \\
c\left(u_{i}, v_{i}\right) & =B & i & =1,2, \ldots, n \\
c\left(u_{i}, w_{i}\right) & =B & i & =1,2, \ldots, n \\
c\left(v_{i}, t\right) & =B+\frac{1}{2} a_{2 i-1} & i & =1,2, \ldots, n \\
c\left(w_{i}, t\right) & =B+\frac{1}{2} a_{2 i} & i & =1,2, \ldots, n .
\end{array}
$$

Define the bound function as

$$
\begin{array}{rlrl}
b\left(s, u_{i}\right) & =0 & i & =1,2, \ldots, n \\
b\left(u_{i}, v_{i}\right) & =0 & i & =1,2, \ldots, n \\
b\left(u_{i}, w_{i}\right) & =0 & i & =1,2, \ldots, n \\
b\left(v_{i}, t\right) & =a_{2 i-1} & i & =1,2, \ldots, n \\
b\left(w_{i}, t\right) & =a_{2 i} & i & =1,2, \ldots, n .
\end{array}
$$

Let $S=\{s\} \cup\left\{u_{i} \mid i=1,2, \ldots, n\right\}$ and $T=\{t\}$.

We claim that there is a feasible even-item partition if and only if there exists a cut $(W, \bar{W})$ covering $(S, T)$ which is a minimum cut with respect to a modified capacity function $c^{*}$ such that $\left|c(e)-c^{*}(e)\right| \leq b(e), \forall e \in A$ and $\sum_{(u, v) \in A} \mid c(u, v)$ $-c^{*}(u, v) \mid \leq B$ 
Obviously, $C^{+}=\left\{\left(s, u_{i}\right) \mid i=1,2, \cdots, n\right\}$ is a minimum cut with respect to $c$, and the capacity of $C^{+}$with respect to $c$ is $\sum_{(u, v) \in C^{+}} c(u, v)=2 n B-\frac{1}{2} B$.

Suppose that there is a cut $(W, \bar{W})$ covering $(S, T)$ which becomes a minimum cut with respect to a modified capacity function $c^{*}$ such that $\left|c(e)-c^{*}(e)\right|$ $\leq b(e), \forall e \in A$ and $\sum_{(u, v) \in A}\left|c(u, v)-c^{*}(u, v)\right| \leq B$.

First since $b(u, v)=0$ for $(u, v) \in C^{+}$, we have $c^{*}(u, v)=c(u, v)$ for $(u, v) \in C^{+}$, and then $\sum_{(u, v) \in C^{+}} c^{*}(u, v)=2 n B-\frac{1}{2} B$. Therefore we have further

$$
\sum_{(u, v) \in(W, \bar{W})} c^{*}(u, v) \leq \sum_{(u, v) \in C^{+}} c(u, v)=2 n B-\frac{1}{2} B
$$

since $(W, \bar{W})$ is minimum with respect to $c^{*}$.

$(W, \bar{W})$ covers $(S, T)$, therefore the non-fixed vertices are $\left\{v_{i}, w_{i} \mid i=1,2, \cdots, n\right\}$. For each $i$, there are three cases:

Case 1. Both $v_{i}$ and $w_{i}$ belong to $W$. Then the corresponding cut arcs are $\left(v_{i}, t\right)$ and $\left(w_{i}, t\right)$.

Case 2. Both $v_{i}$ and $w_{i}$ do not belong to $W$. Then the corresponding cut arcs are $\left(u_{i}, v_{i}\right)$ and $\left(u_{i}, w_{i}\right)$.

Case 3. One of $v_{i}$ and $w_{i}$ belongs to $W$. Then the corresponding cut arcs are $\left\{\left(u_{i}, v_{i}\right),\left(w_{i}, t\right)\right\}$ if $w_{i} \in W, v_{i} \in \bar{W}$, or $\left\{\left(u_{i}, w_{i}\right),\left(v_{i}, t\right)\right\}$ if $v_{i} \in W, w_{i} \in \bar{W}$.

From the bound constraints, only the capacities of arcs $\left\{\left(v_{i}, t\right),\left(w_{i}, t\right) \mid i\right.$ $=1,2, \ldots, n\}$ can be reduced. Denote $x_{2 i-1}=c\left(v_{i}, t\right)-c^{*}\left(v_{i}, t\right), x_{2 i}=c\left(w_{i}, t\right)$ $-c^{*}\left(w_{i}, t\right)$.

Denote $I=\left\{2 i-1 \mid v_{i} \in W\right\} \cup\left\{2 i \mid w_{i} \in W\right\}$.

We have $\sum_{(u, v) \in(W, \bar{W})} c^{*}(u, v)=2 n B+\frac{1}{2} \sum_{i \in I} a_{i}-\sum_{i \in I} x_{i}$.

From (5), we get

$$
\sum_{i \in I} x_{i} \geq \frac{1}{2} \sum_{i \in I} a_{i}+\frac{1}{2} B
$$

By bound constraints, we have

$$
\sum_{i \in I} x_{i} \leq \sum_{i \in I} a_{i}
$$

By the budget constraint, we have

$$
\sum_{i \in I} x_{i} \leq B
$$


Combining (6) and (7), we have $\sum a_{i} \geq B$. Combining (6) and (8), we have $\sum_{i \in I} a_{i} \leq B$. Therefore we obtain $\sum_{i \in I}^{i \in I} a_{i}=B$, that is, $I$ is a feasible even-item partition set.

Conversely, if there is a feasible even-item partition set $I$, let us define a modified weight function as

$$
\begin{array}{rlrlrl}
c^{*}\left(v_{i}, t\right) & =B-\frac{1}{2} a_{2 i-1} & 2 i-1 & \in I \\
c^{*}\left(w_{i}, t\right) & =B-\frac{1}{2} a_{2 i} & 2 i & \in I \\
c^{*}(u, v) & =c(u, v) & \text { all other arcs. } & &
\end{array}
$$

It is straightforward to verify that $\left|c(e)-c^{*}(e)\right| \leq b(e), \forall e \in A, \sum_{(u, v) \in A} \mid c(u, v)$ $-c^{*}(u, v) \mid=B$.

Let $W=S \cup\left\{v_{i} \mid 2 i-1 \in I\right\} \cup\left\{w_{i} \mid 2 i \in I\right\}$. We can easily calculate that $\sum_{w^{\prime}} c^{*}(u, v)=2 n B-\frac{1}{2} B$. It is trivial to show that $(W, \bar{W})$ is a minimum $(u, v) \in(W, \bar{W})$

cut with respect to $c^{*}$ which covers $(S, T)$.

The author is grateful to the Editor and two anonymous referees for their helpful comments which corrected some minor errors of early version of the paper.

\section{REFERENCES}

[1] R.K. Ahuja and J.B. Orlin, Inverse optimization, Part I: Linear programming and general problems, Working Paper, SWP\#4002. Sloan School of Management, MIT, Cambridge, MA (1998).

[2] R.K. Ahuja and J.B. Orlin, Inverse optimization, Part II: Network flow problems, Working Paper, SWP\#4003. Sloan School of Management, MIT, Cambridge, MA (1998).

[3] R.K. Ahuja and J.B. Orlin, Combinatorial algorithms for inverse network flow problems, Working Paper, SWP\#4004. Sloan School of Management, MIT, Cambridge, MA (1998).

[4] M. Cai, X. Yang and Y. Li, Inverse polymatroidal flow problem. J. Comb. Optim. 3 (1999) $115-126$.

[5] M. Cai, X. Yang and Y. Li, Inverse problems of submodular functions on digraphs. J. Optim. Theory Appl. 104 (2000) 559-575.

[6] M. Cai, X. Yang and J. Zhang, Inverse problems with partial given solution, Working Paper. Department of Mathematics, City University of Hong Kong (1997).

[7] M.R. Garey and D.S. Johnson, Computers and Intractability: A Guide of the Theory of NP-Completeness. Freeman, San Francisco (1979).

[8] C. Yang and J. Zhang, Inverse maximum flow and minimum cut problems. Optimization 40 (1997) $147-170$

[9] J. Zhang and M. Cai, Inverse problem of minimum cuts. ZOR-Math. Methods Oper. Res. 48 (1998) 51-58.

[10] J. Zhang and Z. Ma, A network flow method for solving some inverse combinatorial optimization problems. Optimization 37 (1996) 59-72.

to access this journal online:

www.edpsciences.org 